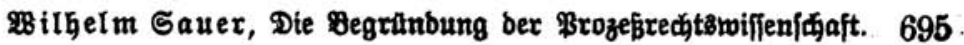

36.

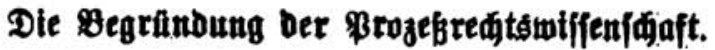

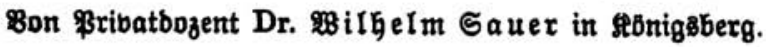

"Die theoretifife $\mathfrak{A r b e i t}$ bringt 'mefr in ber $\mathfrak{B}$ elt zuftande

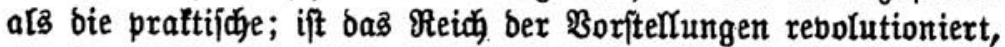
fo bält die Wirflidfleit nidht ftand". - So jeltiam bieje Morte Segels auf ben erften Plid in einer bie Madit ber Tatiadien tiefer benn ie empfindenden Beit anmuten, ihr $\mathfrak{B a h r b e i t 3}$ gehalt ift unberfentbar. Sind bie theoretifhen Brunblagen in baßే :

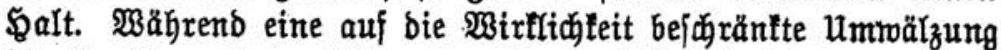

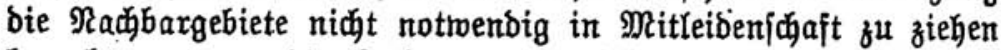

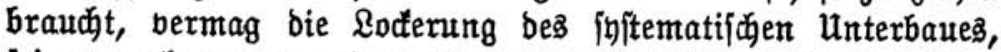
lei ess auch mur an einer einzigen Stelle, bas gejamte wiffens

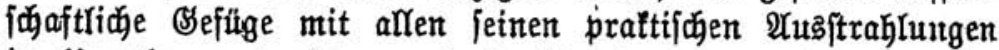
in Unrube zu verjzzen und in Unoronung zu bringen; ein

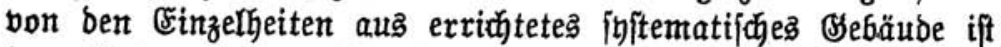
bem. Untergang geroeift, fobalb aud nux für einen einz̧igen, in bem Bau bes Sanzen unentbehrlichen Stein nicht ber note wenbige Raum freigelajfen ift. Wie oft erlebt ber wiffenifiafto liche Foricher bittere Enttäu[dumgen, hat ex nicht von bornherein

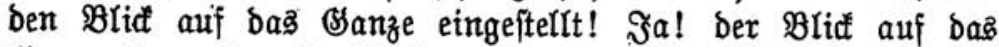
Bsanze ift es, ber bie Mtofaifarbeiten, mögen fie noch jo jauber und reinlich ausgefübrt twerben, exft zu einem harmonijaten,

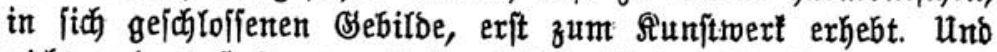
nicht anders fteht es mit ber Wiffenichaft. Die Ridutung auf

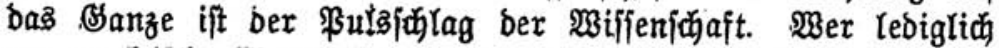
um praftifiche Einzelheiten bejorgt ijt, ber verliert bie F̧üblung

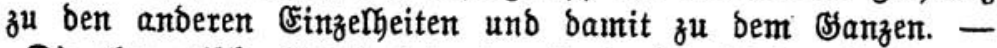

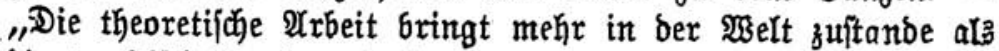
bie praftifiche": eine einzige neue theoretifine Finfidit bermag

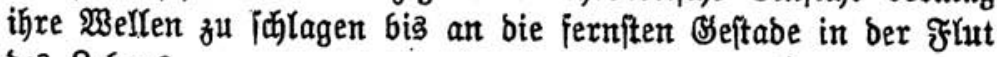
beకె ฉebents.

Retfidr. f. ๖. gef. Strafreditor. XL. 


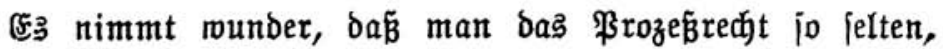
ia eigentlid taum einheitlid =inftematijo als joldes angeidaut hat. Das moderne Bivilprozeß̧recht, oem das reitaus größere Snterefíe ber Syftematifer zugemendet roar, hat man ganz ohne inneren Bufammenhang mit ber Schefterwiffenjhaft des Strafprozeffe: aufgebaut; man hat es pribatredttich ober aud ftaatzred)tlich zu orientieren gejudt. Und dem Strafprozéz andererieitz glaubte man bejonders burdh OUnlehnung an ben

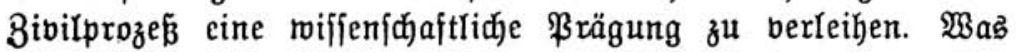

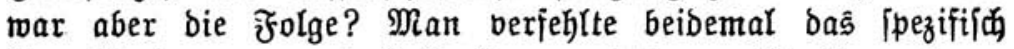
ßrozeffuale; man unterlieś, bie gemeinfamen \$roblemc ftreng

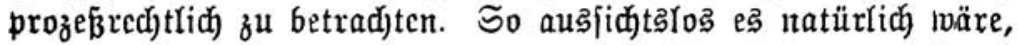

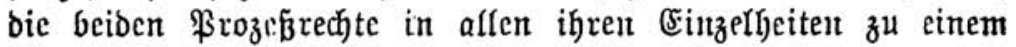
gemeinjamen Suftem zu vereinigen - bazu fint fie benn bod)

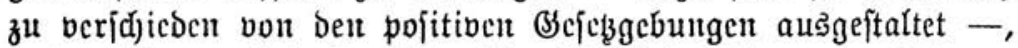

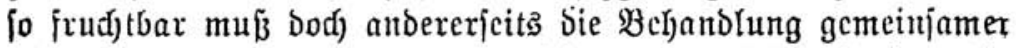
Brobleme unter gemcinjamen Bsefidjt引puntten fein: Sollte nidjt

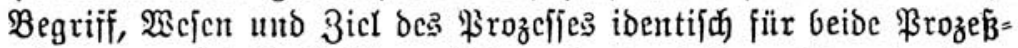
orbnungen jein? Solfte nidyt aud in beiben Brozeßred)ten

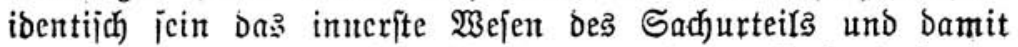

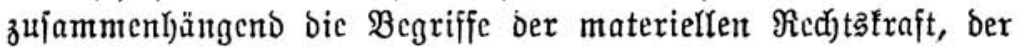
Beweisaufualyme, ber Bollftrectung? Sollten (id) nicht auch

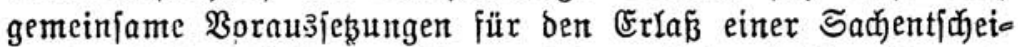
bung aufzeigen laffen, niđjt gemeinfame Arten foldjer Borausెo

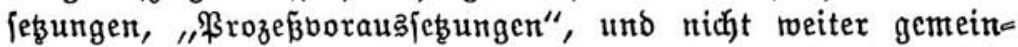
fame Unterarten mit eigenartigen ₹olgen? of vielleidht nidht gerabe ber Błid auj bas sanze eine innerlid gered)tfertigte

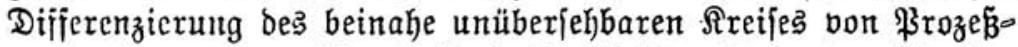

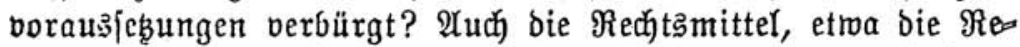
vifion, tragen bod) für beibe ßrozeß̧oronungen gemeinfame Büge zur ธ઼̆au.

Aflcmal bcocutet - bas ift ganz befonders zu beadten -

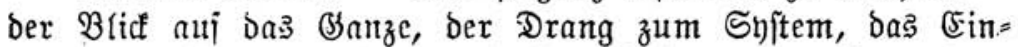
fügen ber Sonberprobleme und ihrer \&öjungen zut einem lyar= monija)en (banzan nicht etroa nur ein (d)ablonenhaftes Dronent bon Eimzclifciten, ctra zur Berwinnutg einer befferen über-

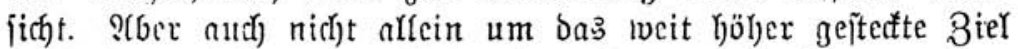
hanbclt e.s iiff, ocm ticf in ber Scele eincs ieden exmíten

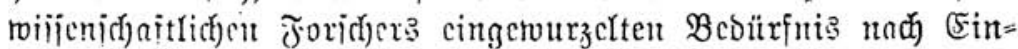

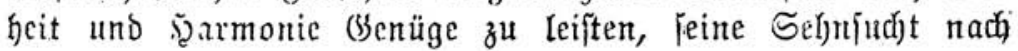


immer und immer reineren und höheren Regionen, immer abjtrafteren Begriffen nad̆ Mäglidifeit zu ftillen, bis man bie Wifferidjaft audh in ihrer Sdjönheit vor fid fieht, bis bas enta zofutte $\mathfrak{A}$ uge ein $\mathfrak{B i l d}$ gerwahrt, in bem alle Einzelheiten und

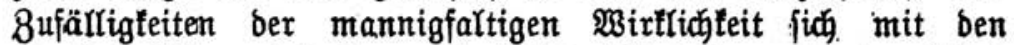

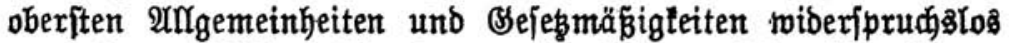

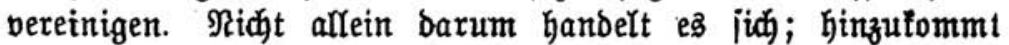
vielmehr noch ein erheblidjer prattifjer Bwect. Das harmoni-

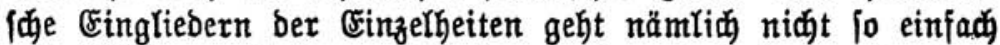
und leidht von ftatten, nidat immer berart, baß̉ bie einzelnen Ergebniffe ohne ieglidje flinberung in bas Sefamtbilb über. nommen werben tönnten; fie müffen nicht felten etras von (iid

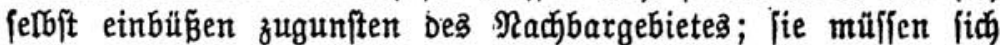

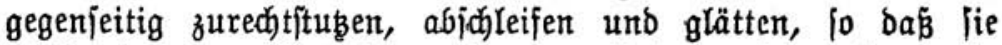
felbfit ein neues $\mathfrak{U}$ usfehen erlangen. Itno bamit geminnen fie

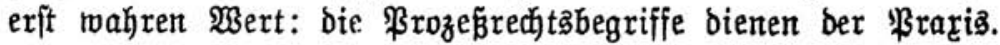
Die Schaffung cines Syftems in unferem Sinne bebeutet baber nicht eine formal=logijøe Sonftruttion - wer heutżutage hätte nod) Freube an einem Derartigen Spiel mit Begriffen! -; ber Sinn eines Syftems ift bielmebr eine Reugeftaltung, eben die Schöpfung des Banzen, bie \$armonie bon Theorie und Praris.

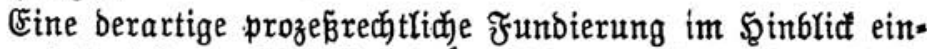
mal auf bie inftematifhe (sinheit und jobann auf bie Bedüriniffe

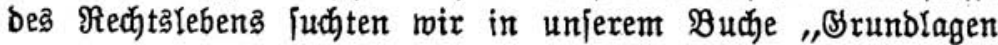

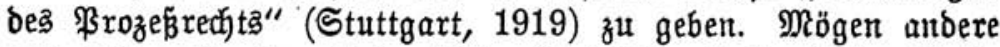
und beffere Werfe mit bemfelben Biel folgen! $\mathfrak{A n}_{\mathfrak{n}}$ biefer Stelle fei es geftattet, einige ßunfte herauşugreifen, mit benen $\mathfrak{u}$. auch unfere Rachfolger werben rechnen müffen, inbem iie zu ihnen in irgendeiner Weife Stełlung zu neljmen haben.

Soll ein prozébrechtlicjes Syftem nicht nur ein Broduft ber \$hantajie bebeuten, fondern auch praftifonen $\mathfrak{B}_{\text {ert }}$ in $\mathfrak{A n}=$

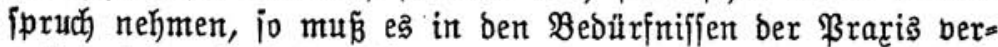
anfert jein. Lthd bas ift ricberum auf bas jichcrite geträhr.

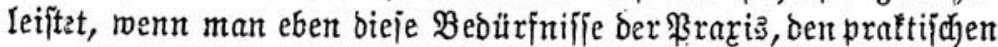

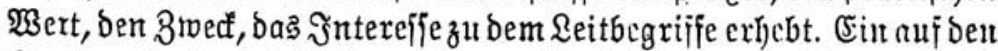
Broce orientiertes Snftem kann nidht lebentafremb jein, und cin Ricuter, ber jeine Enticheidung nach biejem Syjtem trifft, braudht in wenigitens prinzipieller Şinfidyt - anders natürfich), jorncit es itch um die 2lubführung handelt - oen heute fo vit gec 
Görten Bormurf ber Weltfrembheit nicht zu fürdhten, benn ex hat

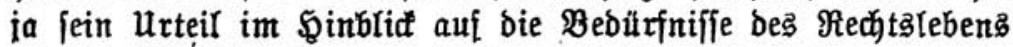

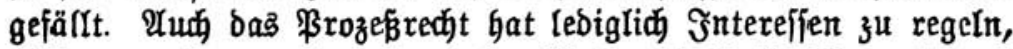

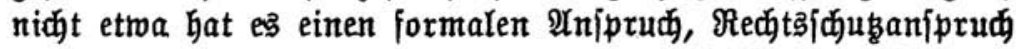
u. bgl. zu realifieren. Und barum gilt es, bieje Эntereffen besె näheren zu beftimmen. Ess gill, bas शrozeß̈recht und jeine

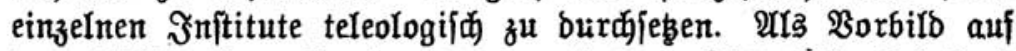
bem Bebiete bes Strafredgts mag bas b. Lisztidje Refrbuch genommen werden: während bort in ben älteren $\mathfrak{A}$ uflagen bei

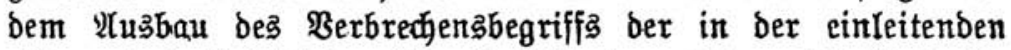

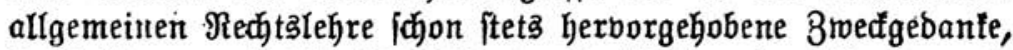

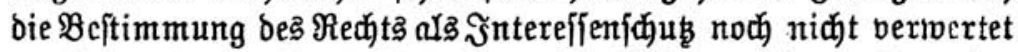
wurbc, während alfo die \&eljrc vom Berbrechen auf b:n allgemein=

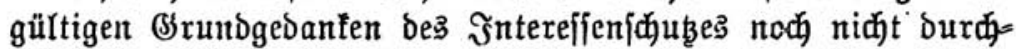
weg eingeftellt war, enthalten bie [päteren Auflagent cine folgec

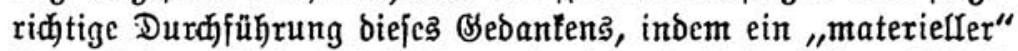

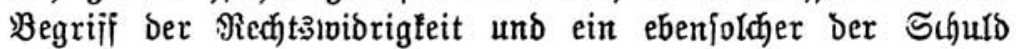
aufgejtellt werben, beren charafteriftifdue Merfmale wejentlich

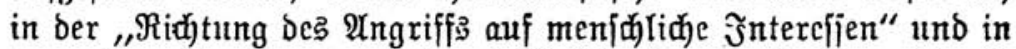

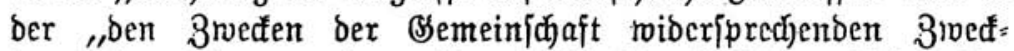

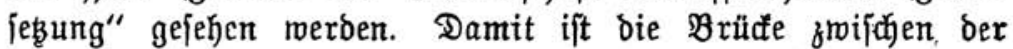

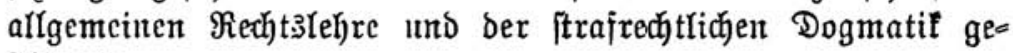
íc)lagen.

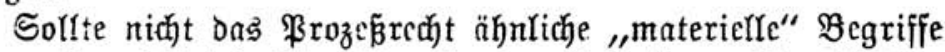

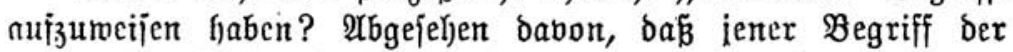

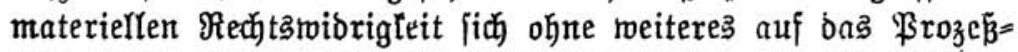

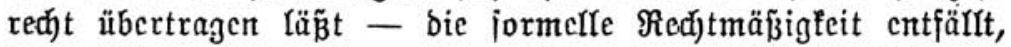

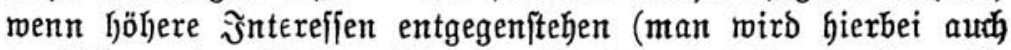
beutlich an ben Belingliben burdfaus materiell gebadten all= gemeinen Rotred)ţ̧egriff crinnert), iie entfüllt $z$. $\mathfrak{B}$. in ben

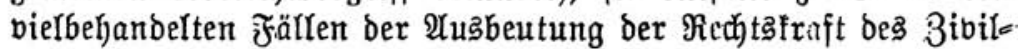
urteiłs miber bie guten Sitten und (durchaus parallel!) in ben

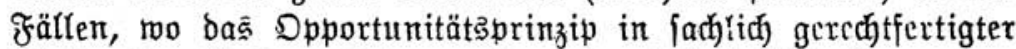
Weife eine Yntlageerhebung oder eine Strafbolrftrecfung ber= bietet - abgejełen bon biejen praftifđ nicht allzu häufigen,

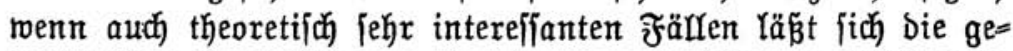

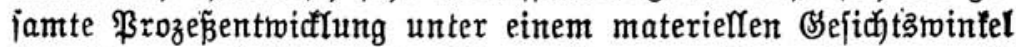
betrachten; und bieje Betrachtung ift notwendig, will man mit

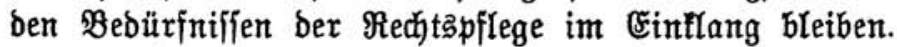

Werdje Intereffen haben benn bie \$rozeß̋beteifigten zu 


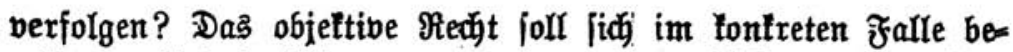
währen; fiat justitia, ne pereat mundus; dieje urteiłämäßige

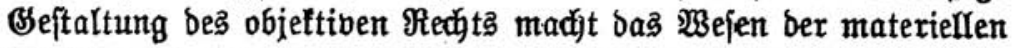

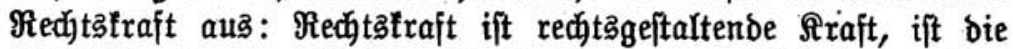

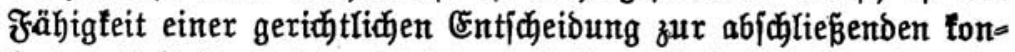

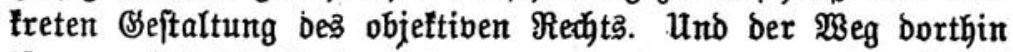

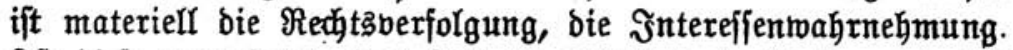

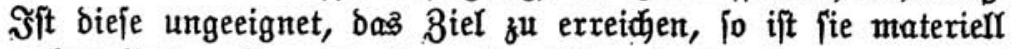

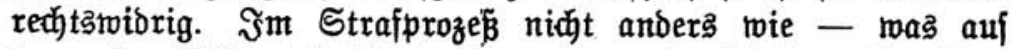

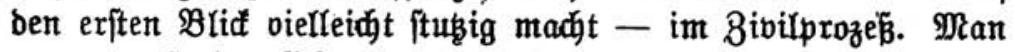

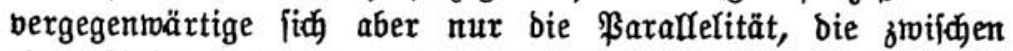

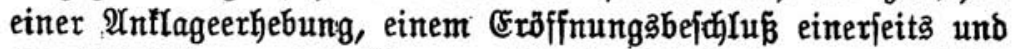
ber Berwilligung bes $\mathfrak{A}$ rmenred ts in Biviljachen andererjeits

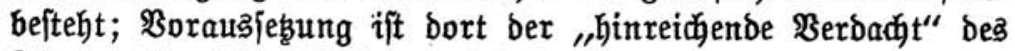

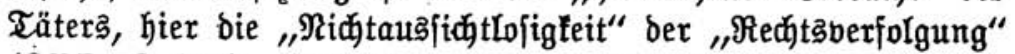
(3RD. § 114). Sadjlid handelt es jid um biejelbe ßoraus= fezung: bie Rechtzoerfolgung barf nidyt ungeeignet jein, bas

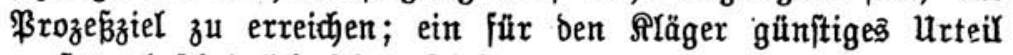

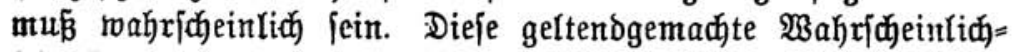

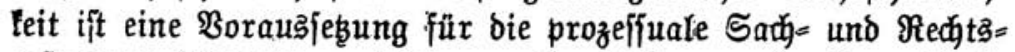
geftaltung (\$rozeß̄oorauşęzung erfter Dronung oder turz: Sad =

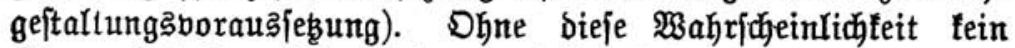

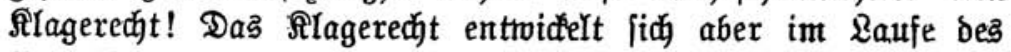

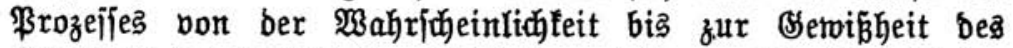

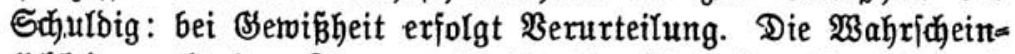

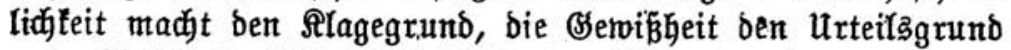

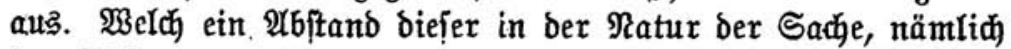

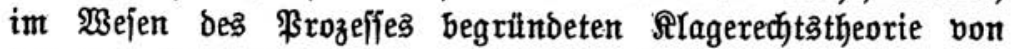

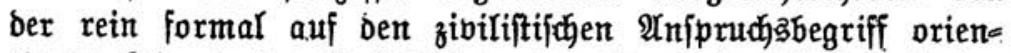

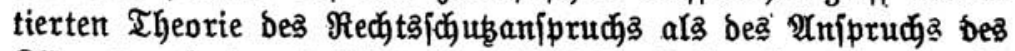

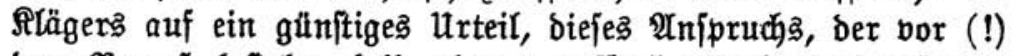

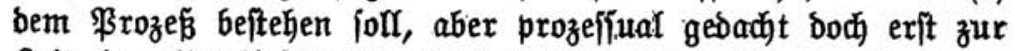

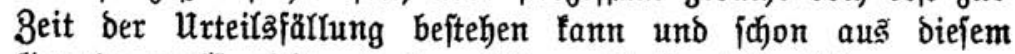

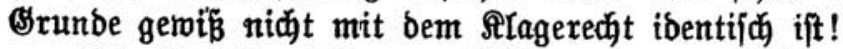

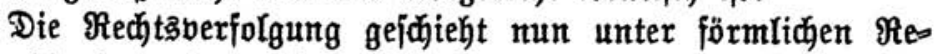

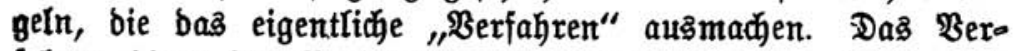
fabren bient ber Berfolgung ebienjo mie bieje ber Sach $=$ uno

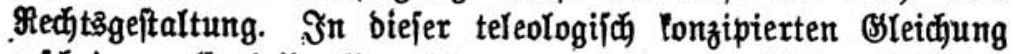

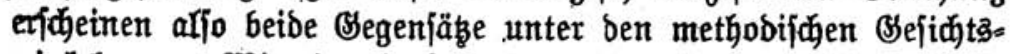

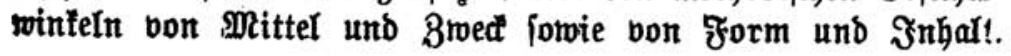


Das förmlidje Berfahren ift das Mittel ber Berfolgung, bieje ift das Mittel ber Sachgeftaltung. So (paltet fin bie Prozeß̧e entridłlung in orei Entridflungsreiłen: Sachgeftaltung, Ber folgung und Berfahren. IYuf allen brei Sinien finden fich ie zrei, einander ourdaus entipredjende ntarfante \$ahrzeidjen:

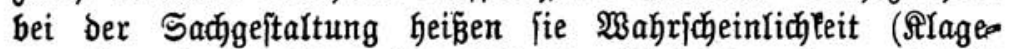

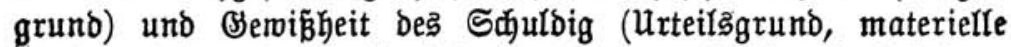

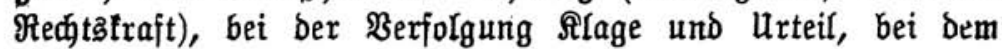

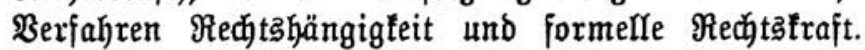

Bezeidnend ift noch cine meitere Bebeutung jener $D i f=$ ferenzierung. Wie bie Sinie ber Sadjgeftaltung an bie er=

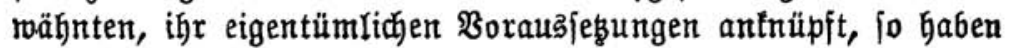
ferner bie Berfolgung und endlich aud das Berfahren ifre

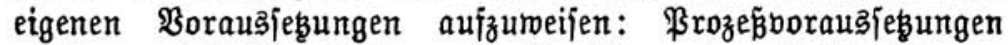
zreiter und britter Dronung ${ }^{1}$ ), Berfolgungs= und Berjahrens=

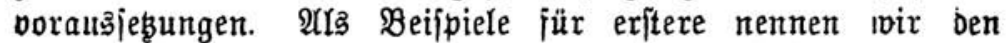
Strafantrag und bas Ridtborliegen eines Shiedşbertrages

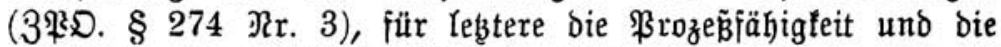

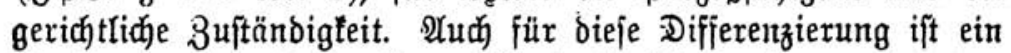

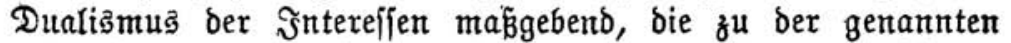

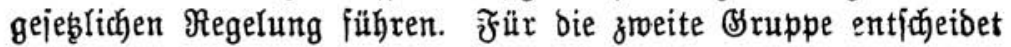

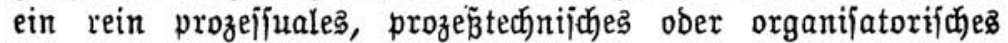

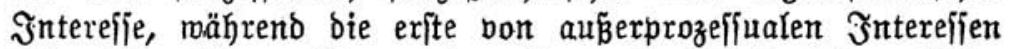

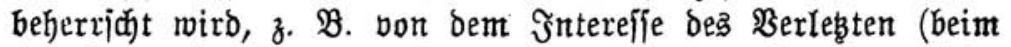
Strajantrag) oder Den \$ribatintereffen ber \$arteien (beim

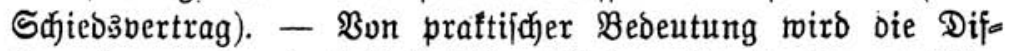
ferenzierung der orei Entridflungşinien aber in ber veriøjiea Denften Weije; io für bie Fragen ber Sceilung und Befebung von Mängeln, der rüđwirfenden Rraft folďer nachträglichen Befebung, ber Bermeiচbarleit ber Mängel, ber Berzichtbarfeit, bes 3citpunttes, an bem bie Boraus]

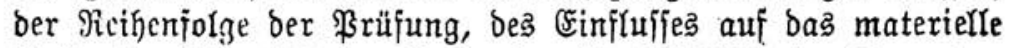

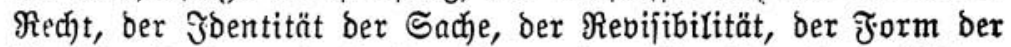
Bemeisaufnahme forie ber f̧orm bes Urteils ufto. Nähere

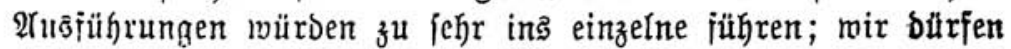
aui unịer oben crröhntes \$erf verweijen. FFs jei nur barauf

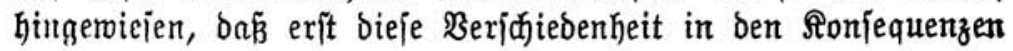

1) $3 u$ einer Mittelgruppe zroijđen ben Sach- unb ben reinen \$rozeßs.

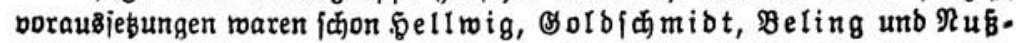
baum gelangt. ફierüber unjer oben angefäbrtes $\mathfrak{W e r t}$ in \& 19 IV. 


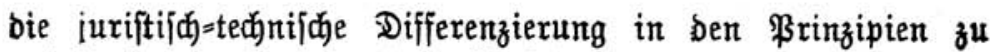
rechtfertigen bermag; würbe fie fehlen, fo räre bie Spaltung praftifid unerkeblidy.

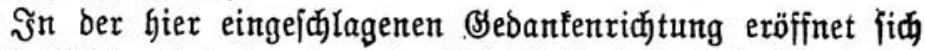

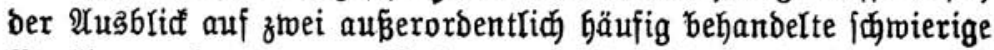
\$robleme, beren enge 8 ujammengehörigfeit und beren eine ander parallele forwic dem $\mathfrak{B}$ ejen ber Sadje entipredjende Röjung einem Borgehen verborgen bleiben mußsten, bas nidgt von born

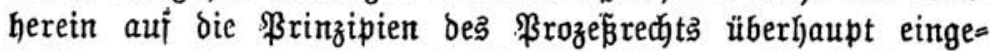
ftellt war. Benau ebenjo wie die Entroifflunģreihen find näm= Iidh aud bie Subjefte einzuteilen in joldse, bie ber Berfolgung, und in joluge, bie bem bloß̧en Berfahren angejören. Nur bie

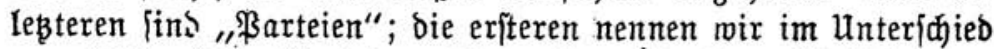
von biejen die "Berfo!gungsintereifenten" ober ,'Berjolgungs= jubjefte" oder "\$rozésinterefienten". Bon beibent Bruppen ver-

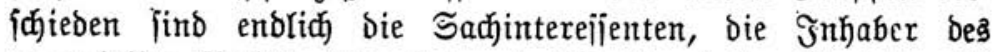
materiellen Rechts. Der Gefannte Streit, ob ber \$arteibegriff ein "materieller" ober "formeller" iít, mirb oon un's alịo bahin entidieden: ex ift ein formeller, ia ein doppelt jormeller; bent bie ßartei fann veriðhieden jein nicht nur oon bem క̃nhaber Dez materiellen Redhts - nur biejen ,formellen" (Eharafter

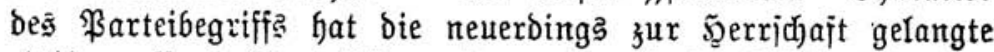

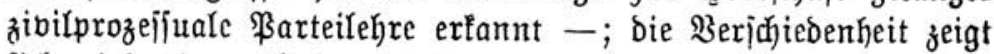
fich bielmehr aud innerhalb dez ßrozeñedhts, hier tritt itod einmal eine Spaltung ein, der ßarteibegrifif gefört nid)t Der

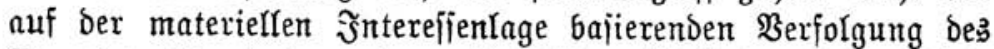

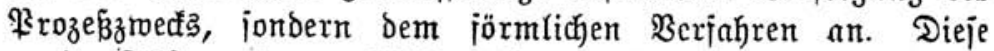
zmeite Differenzierung Glieb völlig unerfantut, und Dodh lag fie fo nahe. $1 \mathrm{~m}$ Dieie Doppelte Formalität an ie einem Beijpiel aus bem Straj= und Bivilprozesrecht zu veranicjaulicjen, lei

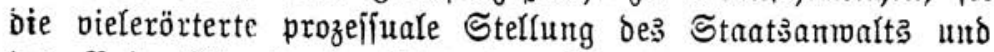

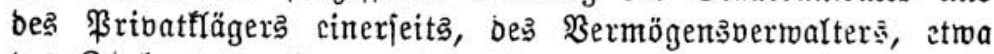

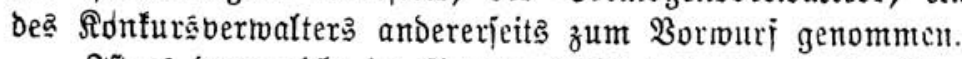

Sfit es benn nicht im Brunbe hödjit miberiintig, im Etraf= prozeñ ben Staat ałz Partei zu betrad)ten, wo er bod über icinte

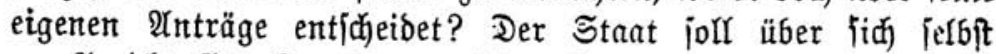
zu Bericht fikgen? und nur feine Bertreter follen beriffieben fein? Dort trete ber Stantäantalt als jein Bertreter (over Srgan) auf, fier bas (Serid)t? Und jerner: ment man ben Staatsantoult mur als \$arteio ertreter bezeidnet, bam muß 


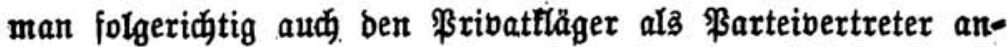
fehen. Wie follte aber ber Staat bazu tommen, fix in Straje fachen burd Pribatperionen vertreten zu Iaffen? Man jebe fid nur einmal jene vor bem Sd̈bffengeridgt berbandelten und

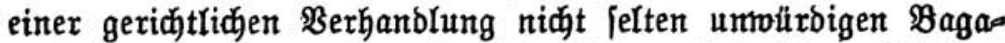
tellfachen an, iene ßrozeffe mit ibrem fleinlidan Sdyimpf

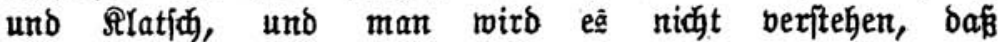
bie ßribatfläger, oft genug reḑt fragmürobige ßerjön= liđffeiten, als prozefiuale Bertreter bes Staatez auftreter. Nein! prozeffual find fie jelbft intereffiert, find fie jelbjt bie ihre prozeffualen Rechte Berfolgenden; ber Staat ift prozefiuar böllig unintereffitert, ein öffentliches ฐ̃ntereffe fehlt, und two es

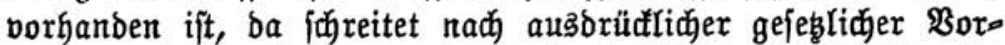

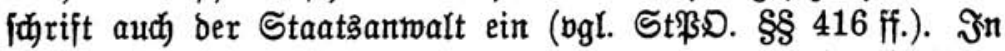
Pribatflagejachen ift ber Staat eben nur materiell red, tli $i$, ঠ. h. als Strafberechtigter, intereffiert, prozeffual auf jeiten

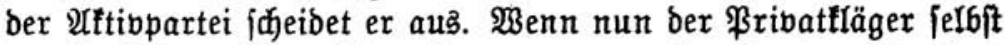
ber Berfolgungsintereffent ift, wenn er - ourchaus analog bem

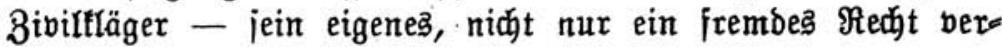

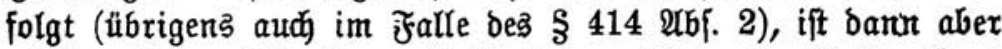
nidht aud ber Staatsెanmalt Berfolgungsintereffent? Er ift es

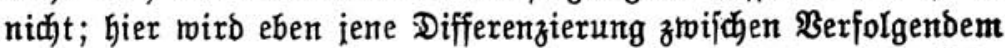
und Partei praftijch. Der Staatsantwalt verfolgt ticht jein eigenes prozeffuales ßecht, jondern ein frembes, ex berfolgt bas offentliche Intereffe; und besెmegen haftet nicht er für bie Soften, ionbern ber Staat, während bort in \$ribatflagejachen burdaus im Eintlange mit unjerem \&runbgebanten ber \$rivata fläger in eigener ßerion ber Softentflidytige ift; beşwegen fommt ferner nicht des Staatzantwalts ßerion in Betradit, wo es

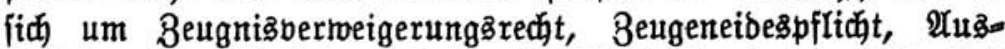

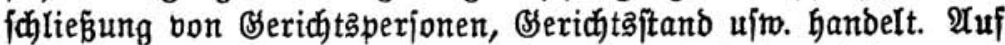
biefe und ähnlidje Boridjriften, bie unzweifelgaft auf ben ma teriell Intereffierten und nicht auf ben vermeintlichen \$rozeß̈bertreter abgeftellt find, hatten twieberholt, und injofern burchaus

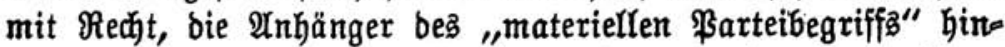

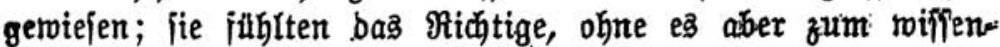

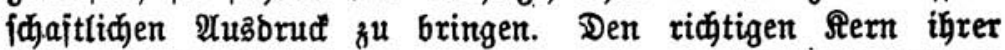
Lehre glaubt bie hier vorgetragene \&rundanjobaunng tarzulegen: iene joeben errähnten Boridriften find auf bie Berfolgenden, bie prozeffualen Intereffenten gemünzt, ßarteien fint 
aber biejenigen, bie in bem jörmlidjen Berfabren ihr Borbringen

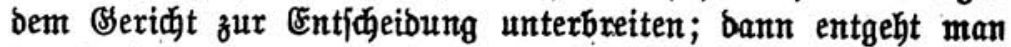

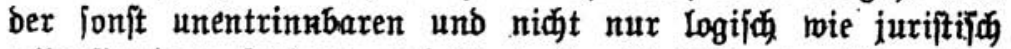

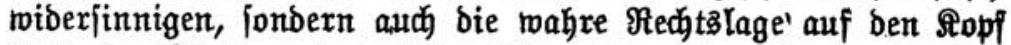

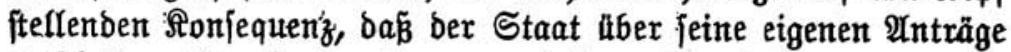
entiøeibe. Um lurz zufammenzufaffen: Sm Dffizials und im

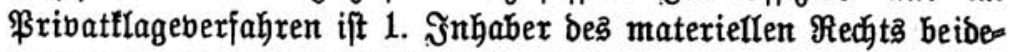
mal der Staat, 2. Berfolgungşıłfieft bort ber Staat, hier ber Pribatfläger, 3. \$artei bort ber Staatsantwalt, bier ber ßribate tläger.

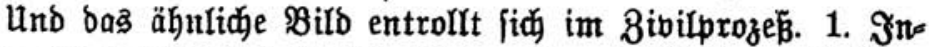

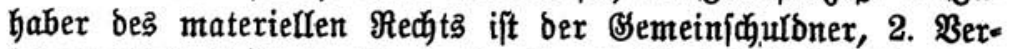

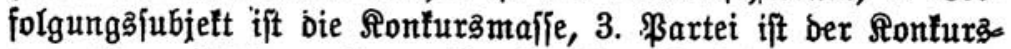

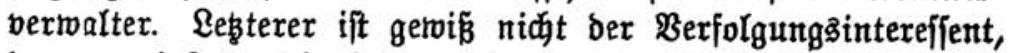
benn er haftet nidyt für bie Roften, nidyt leine eigene ßerjon betreffen bie Boridgriften über ben Beridgtspand, bie Rautionsa

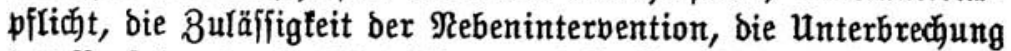
bes Berfahrens. Das hat bie materielle ßarteitheorie (gegenïber ber $\mathfrak{A}$ mtstheorie) burchaus ridtig erfannt. Die genannten $\mathfrak{B e}$. ftimmungen beziehen fich auf bie Sontursmaffe; biefe rieberum tann aber nidjt ßartei fein, will man fie nidjt gar zur jurifti-

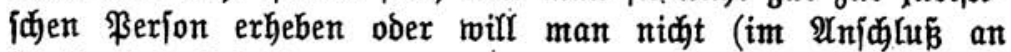
Sellwig Theorie von ben Bermögensmaffen) mit bem Bes fez̧ in Wideripruch geraten, nach bem boch bie felbjtändigen Sonderbermögen teine \$arteifähigfeit genieß̋en. Dẩ anderer-

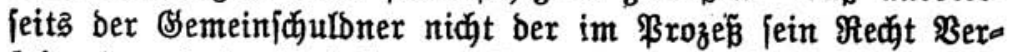
folgende, fonbern daßj prozeffual intereffiert nur ein Teil feines Bermögens ift, bedarf leiner weiteren $\mathfrak{U}$ Haführung; if

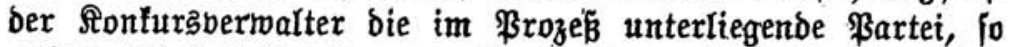

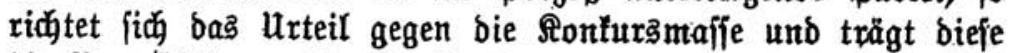

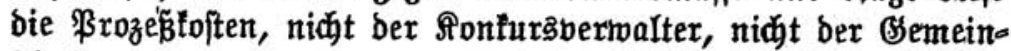
iưuloner.

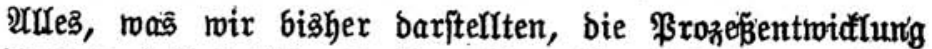

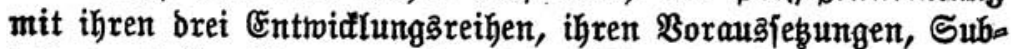
jeften und Şandlungen, alles biejes bilbet bas Dbjett bes \$ro=

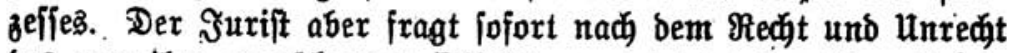
bes bon ing angejuauten Dbjetts, ex betwertet ben Blegenfanto,

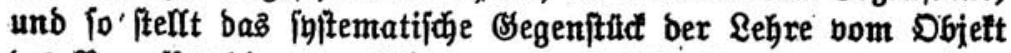

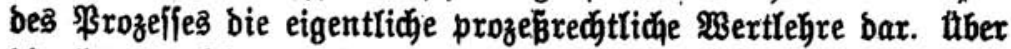
bie leątere fei an biefer Stelle noth ein turzer throerblid geo 
ftattet. Erift odnn vermag man bas હsefügl eines ibftematifđ̣en

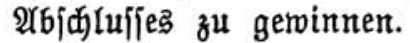

Bier $\mathscr{U}$ rten ber prozeßrechtliçen Bertung find zu unter= ideiben: eine Differnzierung, oie, rohlgemertt, fich mit ber oben burdgeführten Spaltung ber \$rozę̌entroiflung in Die brei Sinien treuzt. Und babei fann gewertet werben alfemal entrober bie gejamte ßrozésentroidlung mit ihren \&inien ober

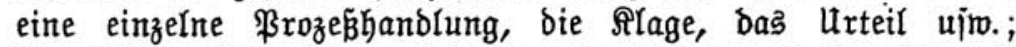

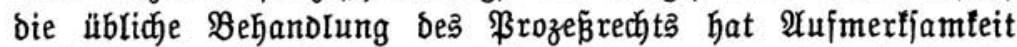
einjeitig bem Urteil ober auch Der §lage zugervendet. Folgende Sdjeibung ift zu treffen. 1. "(s)ültig" ift eine prozeßredhtlidje Ericheinung, went ihre refentrichen Merfmale, ihre Eriftenze bebingungen gegeben jint. Ungültig if́t ein \$rozeß̃ voer eine

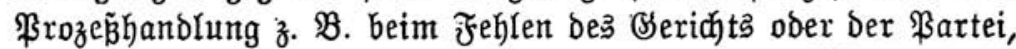
aber aud) bei vöfliger ßartciunfähigfeit. 2. "\$Birtjam" ifft

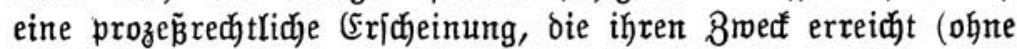

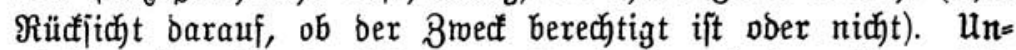

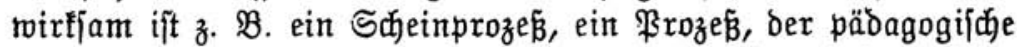

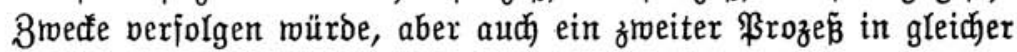
Sache (aljo beim Durd)greifen ber jogenannten Finreden ber

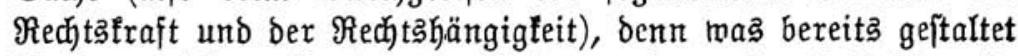

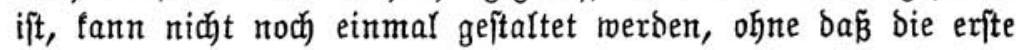
Beftaltung rücfiängig gemacht räre, jo wenig wie ein und basa

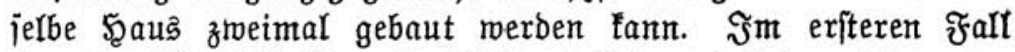

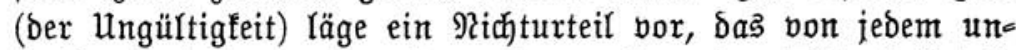
beachtet gelafjen werben tant, im zweiten ein nichtiges urteil, ১as die Begriffzmerfmale eines Urteils tragen tann. 3. "Зu=

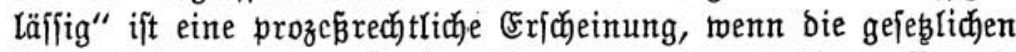
Boraublebungen prozeffualer Ratur gegeben find, z. B. Die

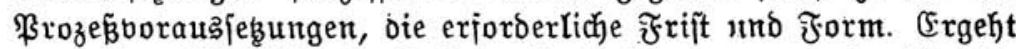

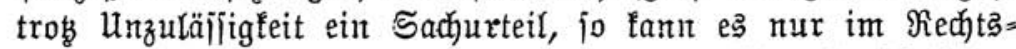
mittelmege aus ber Belt geichafft lverden. Fine Enticheibung aber, bie jich Yediglich) über bie Zuläffigfeit auşpricht (jogenannte

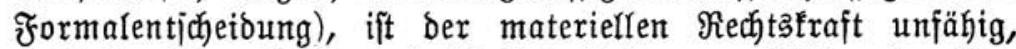

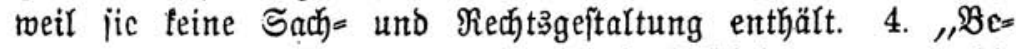
grünbet" entrich ift eine prozeßzred tliche Eridjeinung, went bie

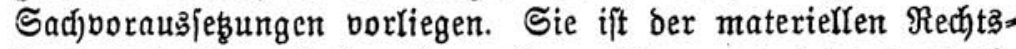
fraft teillfaftig. Ilud anbere, hier nidht zu verfolgende prato rijøe Sonjequenzen ergeben jich aus biejer ßierteilung; mit

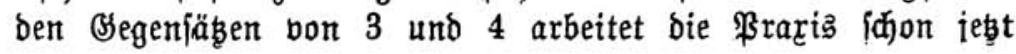


fortmährend, und bas zeigt, bä̉ die Differenzierung praftijळ brauchbar ift. Methobologifd aber ift fie interefiant injofern, als bie ßertítufen 2 und 4 eine fonfrete, Den Brwect einbeziehende

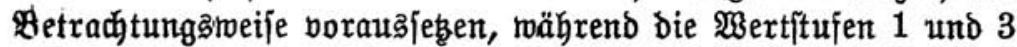
generell, abftralt gebacht find.

Dieje lebstere generelle Betrachtungsart weift aber auf zmei

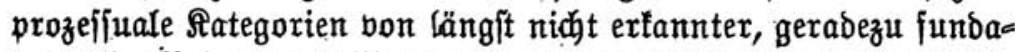
mentaler Bedeutung: Merfmale und Borauşekęungen. Die Mert= male liegen in (!) Dem Ding, Die Boraublekungen bor (!) ihm, vor thm in lugifher, nicht etra in zeitlicher binficht. Das Mertmal ift baher Exiftenzbedingung, die Borauß̧ebung ift 8ulăffigfeitäbedingung. So find, um nur ein Beifpiel anzu-

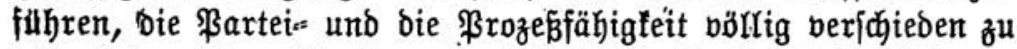
behandeln. Jaene ift ein mejentliches Merimal bes Berfahrens,

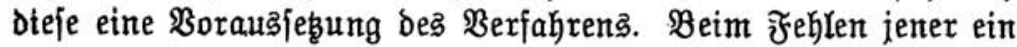

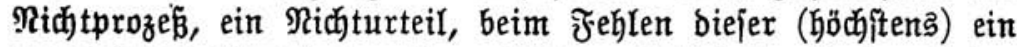

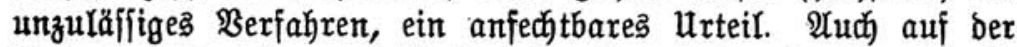
Sinie ber Berfolgung tritt berjelbe Begen[abe auf. Ein Straf= prozeß gegen einen Erterritorialen, ein 8 ibilprozeß gegen ein Sammelvermögen mären eine ungültige ßerfolgung (Nichtber= folgung); beim Fehlen des Strafantrags ober beim Borliegen

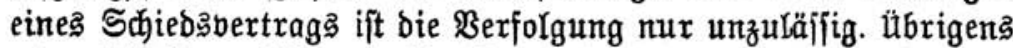
zeigt jich hier wieberum bie Notmendigleit einer Untericheibung von ßerfahren und ßerfolgung in bejonbers hellem Sichte; wir betrachteten ite oben nur auf bem Bebiete der \$rozeß̧poraus= leşungen, fie tritt aber auch bei ben Merfmalen beutlich genug in bie Ericheinung. Die \$arteiunfähigfeit ift zroar bon ber Brozęunfähigfeit veriđhieden, infofern fie ftärfere Wirfungen alā bie Yeştere herbiorruft - basె hatten einige Bertreter ber

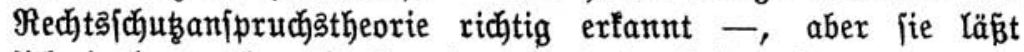
(ich) Doch geriés nicht in einem und bemfelben $\mathscr{U}$ temzuge mit Der Erterritorialität nennen, obroogl jie mit biejer bie fitärfere

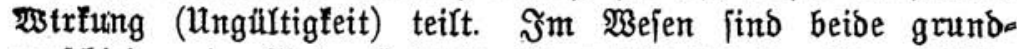

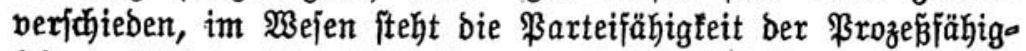

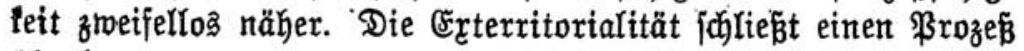
ubberhaupt aus, bie \$arteiunfähigfeit nur eine gewiffe Frorm

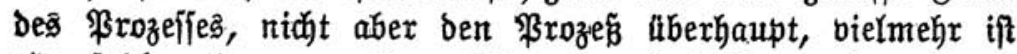
ein poldher bann z. $\mathfrak{B}$. nicht gegen bas Sammelvermögen, fondern gegen einen bejonders beftellten \$fleger zu führen. Und fo ipringt die tiefgebende $\mathfrak{B e j e n z b e r i d i e b e n h e i t ~ b o n ~} \mathfrak{B e r}$ 


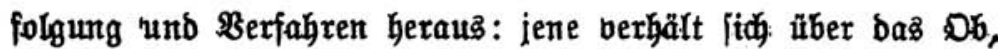

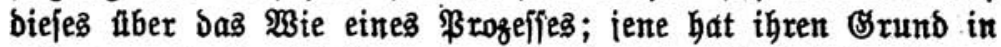

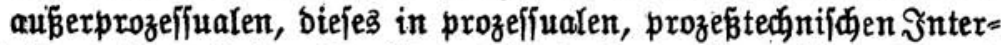
effen. Itber biejen Dualismus barf aber nidjt außer adyt ges Iaffen werben ber andere zulez̧t behandelte Bsegenfaz von Bor auşęung und Mertmal, bex prinzipieffer erfaß̧t zu unjerer oben bargeftellten ßierteilung ber pnozeßrechtlichen Wertung hin= fithrt.

Bielleidyt hat bie eine ober bie andere, viefleidyt haben aud

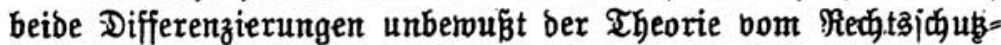

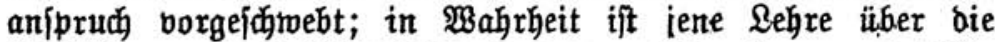

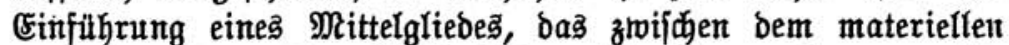

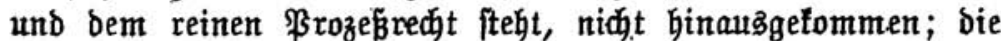
Differenzierung Der Borauşęzungen (im weiteften Sinne) unઠ

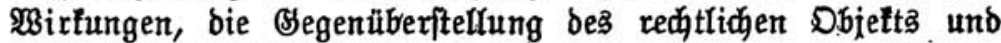
bes rechtlichen Maß́f́tabs und bamit bie Preuzung Der Bsegen

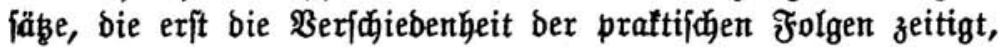

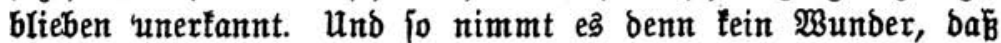

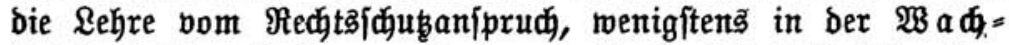
Sellwiginen Faffung, als ein rein tonftruttibes Bsebilde ohne jebe praftijage Bebeutung auftritt. Wie bezeidynend ift es bod, Daß̧ unjer herborragender Sommentator ber Bibilprozeß̧oronung,

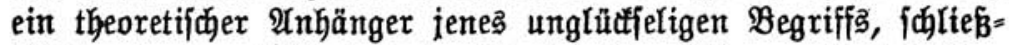

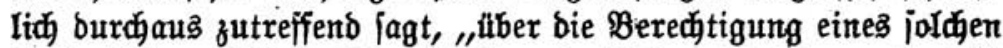
lonftruttiben Behelfes" entifheibe aim Ende "bie Berwertbarteit bes Begriffes für bie Crkenntnis und bie prattifide Antrentoung bes geltenden Red $t^{\prime \prime \prime} !^{2}$ ). Und wie bezeidjnent, went er fid

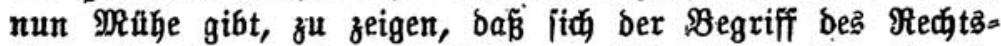

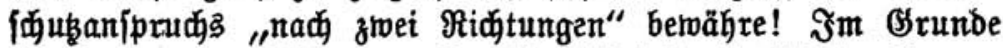
ift es nur eine Ridutung. Der Begriff foll nämlid, im Elegen-

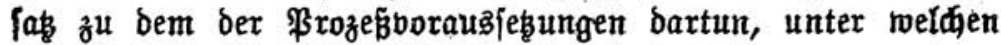

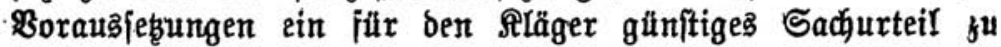
exlafien ifit. Diefen Borauşjęzungen ift freilich eine eingebenbe

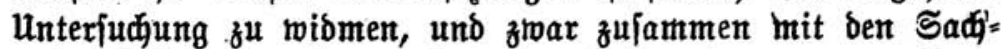

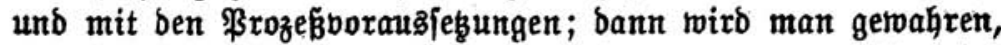

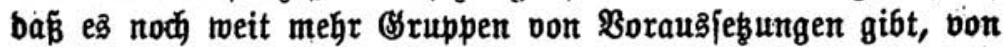
benen eine jebe bon ber anderen forohl in ihrem Befen wie in ifren \$irhungen berifiteden ift. Erft bamit ift bie theoretijue wie prattiface Exiftenzberedigung einer begriffliden Spalturig

ๆ) Stein, 8æD., 11. צufl., 1913, vor \& 253 III थbf. 2. 


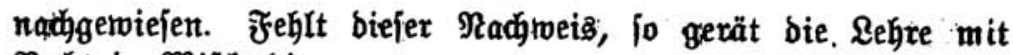
Recht in Míptrebit.

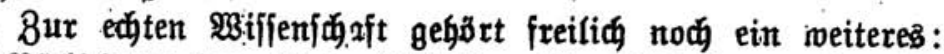

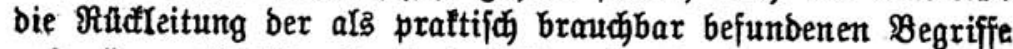

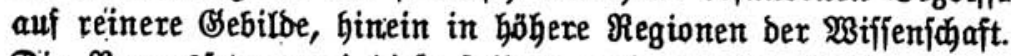

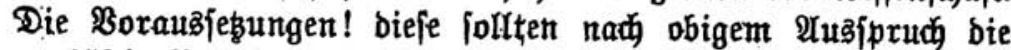

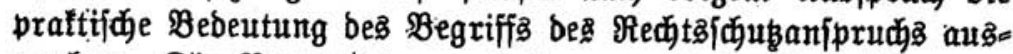
madjen. Die Borausijekungen! $\Im a$, um biejen Begriff zu ge= winnen, bedurfte es rahnlich nicht ber Einführung bes zibilifti-

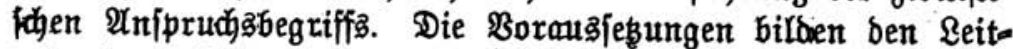

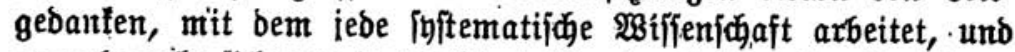
man braudit fich mur in bie Borballen ber Rehren ber Rantianex

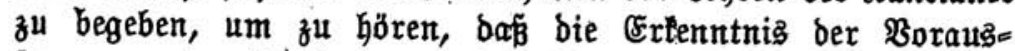
fę̧ungen ober Bebingungen bas \$ejen ber \$iffenifdaft itber

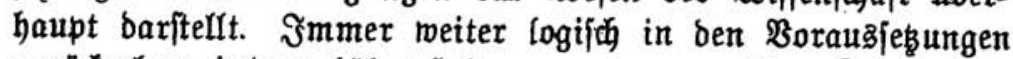
zurüfoheh, immer böher fteigen, zu immer allgemeineren $\mathfrak{B} e$

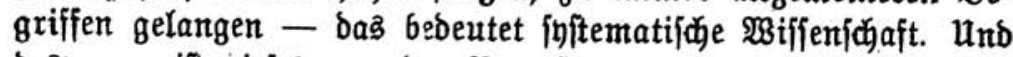

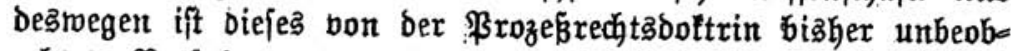
ad) tete Berfahren einzujiflagen, will man bas \$rozeß̧recht aโsె

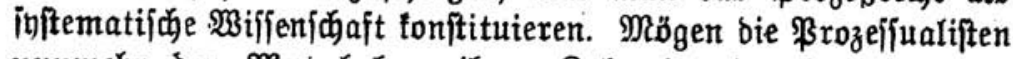
nunmehr ben Mut yaben, ihren $\mathbb{R a h n}$ in ein nie befahrenes Waffer zu lenten!

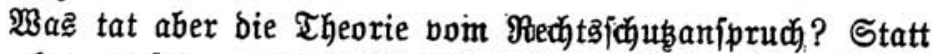
nach oben zu fehen, wandte iite fid. nach der Seite; fie entlehnte

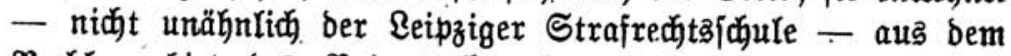

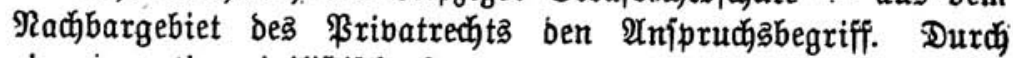

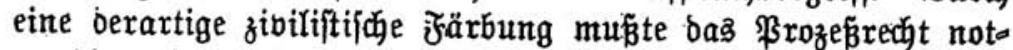
wentig einen indivibualiftificen uno zugleidy frembartigen Eharafter anrebmen. Sene Rehre fterlt fich nicht auf ben Stando punlt bes übergeoroneten (Serichts, fonbern auf ben ber \$arteien,

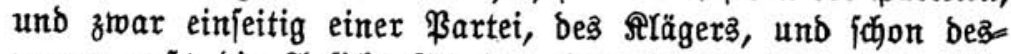

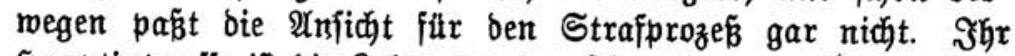
Sauptintereffe ift bie Sefre von ber ßage (man werfe nur einen

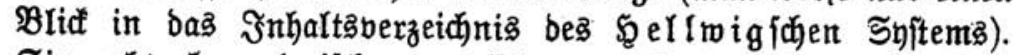
Sic geht dronologif bor. Db es nicht ber Errfenntnis bes Wejens fïroerlicher ift, nach bem 3 iel zu fragen? nadj bem Brwed und Sinn bes \$rozeffes? Unter biejer Einfteflung wirb

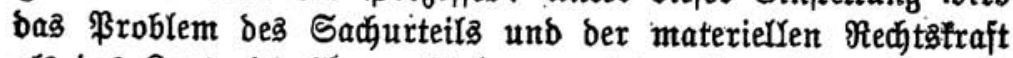
als bas Bentralprobłem erijheinen, und ber Begriff ber $\mathfrak{B o r a u b s}$

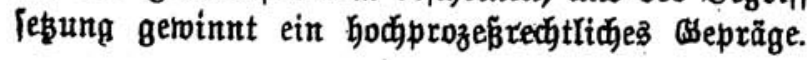




\section{8 ஐ}

und if andererjeits ber bier beidurittene Pfad nidyt aud

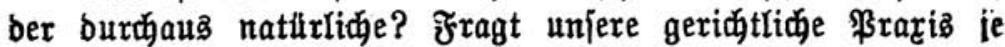

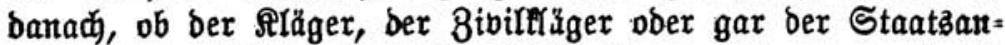

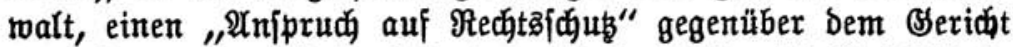

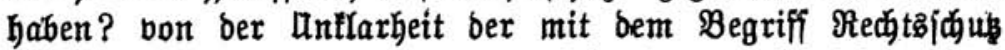
verbunbenen Borftellung ganz zu jurveigen. Ş nidt natule licher bie jolgenbe Betrachtung? Die \$rozeßplubiefte fragen: wna will ich erreichen? wie berfolge und geftalte ich bas Recht?

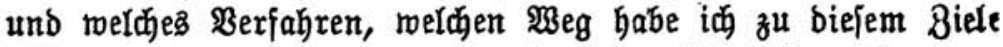
einzufichlagen? 8u biejem Begenftand bes. Prozefiez tritt bann bie \$ertung auf recht und unrecht. Und in biejer Sinficht in

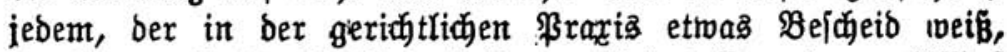
burđaus geläufig bie ₹rrageftellung: ift bas Borgehen zuläfifig? und jobann bie meitere: ift es begründet?

Dbjeft und ßert! : 8u biejen oberften, der alfgemeinen

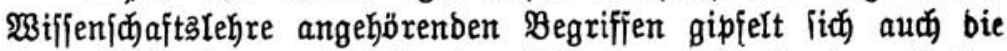

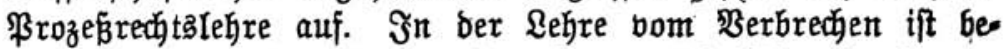

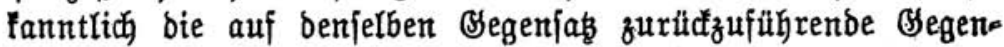

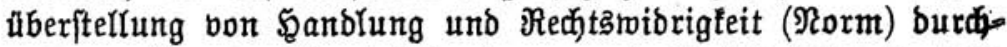
aus anerfannt. $\mathfrak{B}_{0}$ türbe bagegen robs bie Theorie vom

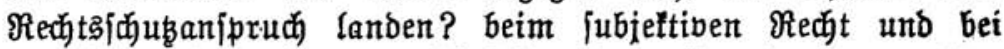
ber Befugnis? Raum wohl beim Sntereffe; benn zu einer "materiellen" Finftellung befteht bei ben Shülern und Nadg. folgern $\mathfrak{B}$ indideids feine Neigung. Sdjwerlich aber, io rie es jein follte, beim objeftiven Reḑt, beim Máfítab ber Wertung.

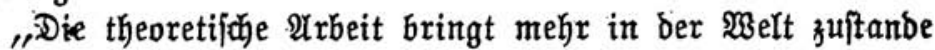
als bie praftifude." Diejen unjeren Eingangstworten jei ber Wunjch hinzugefügt: Möge ber Shjtematifer nach biejer Err= Penntria um io eifriger bemulht fein, ber \$raris zu nüben! Dann wirb bie \$raria jich nach ber Theorie "ridenten", nach bem Theoretifer als bem wahrhaften Ridjter. Dann wirs bas bohe

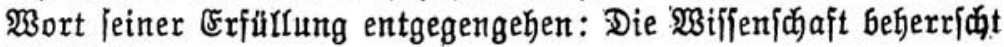
bie $\mathfrak{\text { Beít. }}$ 